Original Paper http://ajol.info/index.php/ijbcs http://indexmedicus.afro.who.int

\title{
Effet comparé de la température sur quelques paramètres biologiques de deux biotypes de Maruca vitrata Fabricius (Lepidoptera : Crambidae) en condition de laboratoire
}

\author{
Fousséni TRAORE ${ }^{1 *}$, Antoine WAONGO ${ }^{1}$, Clémentine DABIRE-BINSO ${ }^{1}$ et \\ Antoine $\mathrm{SANON}^{2}$ \\ ${ }^{1}$ Laboratoire Central d'Entomologie Agricole de Kamboinsé, Institut de l'Environnement et de Recherches \\ Agricoles, Burkina Faso. \\ ${ }^{2}$ Laboratoire d'Entomologie Fondamentale et Appliquée, UFR/SVT, Université Ouaga I Pr Joseph KI-ZERBO, \\ Burkina Faso. \\ *Auteur correspondant ; E-mail : foussnitraore@gmail.com
}

\section{RESUME}

Maruca vitrata est un ravageur des gousses de niébé qui vit en cycle continu dans l'agrosystème du Burkina Faso où les températures moyennes sont élevées et le taux d'humidité relativement bas. Comment l'insecte est-il parvenu à s'y maintenir alors que son écologie requiert des températures modérées et de forte humidité ? La réponse à cette interrogation a motivé la conduite de cette étude dont le but est de comparer l'effet de cinq températures sur quelques paramètres biologiques du biotype du Burkina, et du celui du Bénin utilisé comme témoin. Les résultats ont révélé que la ponte moyenne du biotype du Burkina est significativement plus élevée que celle du biotype témoin. Les taux d'éclosion des œufs du biotype du Burkina à $32^{\circ}$ et $34^{\circ} \mathrm{C}(\mathrm{P}=0,0078$ et $\mathrm{P}=0,0003)$ sont plus élevés que ceux du témoin. Les mâles du biotype du Burkina ont une durée de vie plus élevée $(\mathrm{P}=0,02)$ seulement à $34^{\circ} \mathrm{C}$ que ceux du témoin tandis que chez les femelles, elle l'est à $25^{\circ}, 30^{\circ}, 32^{\circ}$, et $34^{\circ} \mathrm{C}(\mathrm{P}=0,42 ; \mathrm{P}<0,0001 ; \mathrm{P}=0,02 ; \mathrm{P}<0,0001)$. L'insecte se serait adapté à l'environnement du Burkina et pourrait être différent du biotype du Benin.

(C) 2018 International Formulae Group. All rights reserved.

Mots clés : Maruca vitrata, biotypes, températures, paramètres biologiques.

\section{Compared effect of the temperature on some biological parameters of two strains of Maruca vitrata Fabricius (Lepidoptera: crambidae) under laboratory condition}

\begin{abstract}
Maruca vitrata is a damaging pest of cowpea pods that lives throughout the year in the agro-ecosystem of Burkina where average temperature is high and relative humidity low. How did the insect succeed to cope this environment while its ecology requires moderate temperatures and high humidity? The response to this question motivated this study which aimed at comparing the effect of five temperatures on some biological parameters of Maruca strain from Burkina to those from Benin used as control. The results showed that the average laying of the strain from Burkina is significantly higher than that of the control. The hatching rates of
\end{abstract}


the strain of Burkina at $32^{\circ}$ and $34^{\circ} \mathrm{C}(\mathrm{P}=0.0078$ and $\mathrm{P}=0.0003)$ are higher than those of the control. Males of the Burkina's strain had longer lifespan $(\mathrm{P}=0.02)$ at $34^{\circ} \mathrm{C}$ than that of the control, whereas for the females it was higher at $25^{\circ}, 30^{\circ}, 32^{\circ}$ and $34^{\circ} \mathrm{C}(\mathrm{P}=0.42, \mathrm{P}<0.0001, \mathrm{P}=0.02, \mathrm{P}<0.0001)$. The insect would have adapted to the environment of Burkina and could be different from the strain of Benin.

(C) 2018 International Formulae Group. All rights reserved.

Keywords: Maruca vitrata, strain, temperatures, biological parameters.

\section{INTRODUCTION}

Le niébé [Vigna unguiculata (L.) WALP.] est une légumineuse alimentaire largement cultivée dans le monde avec une production estimée à 6,4 millions de tonnes (FAOSTAT, 2013) de graines sèches. La production de l'Afrique subsaharienne représente environ $95 \%$ de la production mondiale de niébé dont plus de $80 \%$ est produite en Afrique de l'Ouest (Sanginga et Bergvinson, 2015). Produit essentiellement en pluviale pour ses vertus économique, sociale et alimentaire, la production actuelle du niébé demeure faible pour couvrir les besoins alimentaires nationaux et la demande sous régionale sans cesse croissante. Les rendements graines actuels du niébé sont de 700 à $800 \mathrm{Kg} / \mathrm{ha}$ en culture pure avec des variétés améliorées (Kaboré, 2013) comparativement au rendement potentiel de la plante qui est estimé entre 1,5 et 3,0 t/ha (Downham et al., 2003). Plusieurs contraintes dont les insectes sont à l'origine des faiblesses de rendements car à chaque stade du cycle de développement de la plante, au moins un insecte majeur peut causer des dégâts considérables et réduire la production (Singh et Jackai, 1985). Maruca vitrata Fabricius (Lepidoptera: Crambidae) est un ravageur important du niébé dans les régions tropicales et subtropicales humides.

Dans la partie ouest du pays à pluviométrie plus élevée, $M$. vitrata a été répertorié comme un ravageur important avec les dégâts qui sont variables d'une saison à l'autre. Ces dégâts qui restent endémiques dans toute la région (Dabiré, 2001 ; Ba et al., 2009) sont dus aux larves qui s'attaquent aux boutons floraux, aux fleurs et gousses du niébé (Traoré et al., 2013) occasionnant des pertes de production estimées entre 20 et $80 \%$
(Atachi et al., 2002). La production durable et rentable du niébé fait donc de la lutte contre ces insectes ravageurs une impérieuse nécessité (Kadri et al., 2013).

Cependant, il est quasi impossible de lutter contre un insecte ravageur sans une connaissance de son écologie. Cette écologie inclut la dynamique des populations adultes et larvaires ou le suivi de l'évolution des effectifs, l'inventaire des parasitoïdes et des plantes hôtes de l'insecte. Les études déjà menées ont révélé que dans les zones humides, les populations de M. vitrata se développent dans les agro systèmes, en cycle continu sans diapause (Traoré, 2014). L'insecte transite par une série de plantes hôtes de relais au cours des saisons sèches et infeste les cultures de légumineuses du genre Vigna dès la reprise de la saison des pluies. À la vue des conditions climatiques peu favorables, les populations adultes de $M$. vitrata migreraient des zones humides vers la zone sahélienne où elles font une seule génération, puis migrent vers le nord où les populations se perdent, ne pouvant survivre aux conditions rudes de l'environnement (Adati et al., 2013).

Dans ce contexte de supputations et d'hypothèses, des études portant sur quelques éléments d'écologie de ce ravageur ont été menées par Traore et al. (2014). Elles ont abouti à la conclusion que l'insecte vit et maintient ses populations sur des plantes hôtes durant toute l'année à l'ouest du Burkina Faso (Traoré et al., 2014). En effet, pendant qu'aucune capture d'insecte adulte n'a été enregistrée au piège durant la saison sèche, des populations résiduelles constituées de larves ont été collectées durant toute l'année sur des plantes hôtes alternatives au sud-ouest du Burkina Faso, pays sous climat peu 
humide, aux températures relativement élevées et aux plantes peu abondantes. Dès lors, une question taraude l'esprit, celle de comment $M$. vitrata parvient à se maintenir dans un environnement aux conditions écologiques peu favorables, alors que sa biologie et son écologie sont tributaires d'un taux d'humidité moyen de $70 \%$ et de basses températures d'environ $25^{\circ} \mathrm{C}$ (Atachi, 1998). C'est la recherche de la réponse à cette question qui a motivé la conduite de la présente étude dont l'objectif est de tester et comparer l'effet de la température sur quelques paramètres biologiques des populations du biotype du Burkina en comparaison avec celui du Bénin. En effet, la température est un des plus importants facteurs qui affectent fortement la croissance et plusieurs paramètres du cycle de vie (Steigenga et Fischer, 2009).

\section{MATERIEL ET METHODES}

\section{Site du test}

L'étude s'est déroulée dans le centre de lutte biologique de IITA-Cotonou. L'effet de la température sur quelques paramètres biologiques du biotype du Burkina a été étudié en comparaison avec ceux du biotype du Bénin.

\section{Obtention des insectes}

Les insectes du Burkina ont été prélevés dans les élevages de masse au laboratoire. Ces insectes, auparavant, ont été mis en place à partir des individus sauvages capturés au piège lumineux dans le mois d'août à Farako-Bâ dans l'ouest du pays. Ils ont été maintenus en élevage sur substrat artificiel en condition de laboratoire (Jackai et Raulston, 1988). Les températures et le taux d'humidité dans la zone de capture étaient respectivement de l'ordre de 20 à $38^{\circ} \mathrm{C}$, de 60 à $91 \%$. La pluviométrie annuelle enregistrée durant la période de capture était de $1200 \mathrm{~mm}$ d'eau.

Le biotype de Cotonou a été prélevé de l'élevage de masse de l'Institut International d'Agriculture Tropical (IITA), station de Cotonou au Benin. Ces insectes étaient maintenus sur le substrat artificiel dans le laboratoire du centre de lutte biologique. Le substrat utilisé est préparé selon la méthode décrite par Jackai et Raulston (1988).

Deux cent cinquante (250) chrysalides de chaque biotype ont été utilisées comme insectes «stock» de départ pour les études. Le biotype de Benin a constitué le témoin en raison de sa provenance.

A l'émergence, les adultes ont été maintenus de façon séparée dans les cages faits en plexiglas de dimension $20 \mathrm{~cm} \times 15 \mathrm{~cm}$ $\mathrm{x} 15 \mathrm{~cm}$ pour accouplement durant quatre nuits consécutives. A l'issue de ce temps d'accouplement, les femelles ont été isolées et placées par ratio de trois dans les tubes pour la ponte. Les œufs ont été collectés 24 heures après la mise en oviposition et placés dans la salle d'élevage des adultes. A l'éclosion, une partie des larves de chaque biotype est placée sur le substrat pour la continuité de l'élevage de masse, tandis que l'autre moitié a été utilisée pour les tests.

\section{Conduite des tests}

Les températures de $20^{\circ}, 25^{\circ}, 30^{\circ}, 32$ o, et de $34{ }^{\circ} \mathrm{C}$ ont été retenues pour conduire les tests. Ces températures sont obtenues par réglage à l'intérieur de chaque incubateur jusqu'à la fin de l'étude. Des bacs en plastique de capacité 3 litres ont été remplis d'une quantité de 2,5 litres d'eau et déposés à l'intérieur de chaque bio incubateur, comme source d'humidité. L'eau était rajoutée à chaque fois que la quantité de celle-ci diminuait.

\section{Effet de la température sur le taux de mortalité et l'émergence des adultes}

Le substrat artificiel a été préparé et coulé dans les boîtes transparentes de $250 \mathrm{ml}$ de capacité. La quantité de substrat coulée dans chaque était de $150 \mathrm{ml}$. Lorsque ce substrat se solidifie, sa surface supérieure a été scarifiée à l'aide de fourchette stérilisée avec de l'alcool $90^{\circ} \mathrm{C}$ pour éviter des contaminations et faciliter la prise de la nourriture par les premiers stades larvaires. Pour l'infestation, les larves néonates ont été reversées sur du papier blanc et reprise une à une à l'aide de pince souple pour être déposée 
sur le substrat. La boîte est d'abord refermée à l'aide du papier essuie-tout ensuite de la toile mousseline et enfin du couvercle portant une ouverture circulaire. Ainsi, cent (100) larves par biotype ont été placées sur le substrat en cinq répétitions, soit un total de cinq cents (500) larves par biotype et par température. Le substrat infesté de larves est placé dans un incubateur et la température est réglée. Pour chaque température testée, quatre répétitions ont été constituées. Les observations ont consisté au dépouillement et au dénombrement des larves juste avant que celles-ci n'entrent en phase de chrysalidation. A l'émergence, les adultes ont été également dénombrés.

\section{Effet de la température sur la durée de développement larvaire}

Les flacons transparents de capacité de $30 \mathrm{ml}$ chacun et contenant $15 \mathrm{ml}$ de substrat ont été utilisés dans l'étude des stades larvaires. Deux larves néonates ont été placées par flacon contenant du substrat en cinq répétitions. Le développement des larves a été suivi juste avant leur entrée en diapause. A cette étape, les larves ont atteint le stade cinq (larves de couleur verdâtre). Elles sont soigneusement extraites à l'aide de pinces souples et gardées dans des flacons transparents de $30 \mathrm{ml}$ jusqu'à la formation de la chrysalide. La durée moyenne de développement larvaire a été calculée. Elle va du stade 1 jusqu'au moment où la larve cesse de s'alimenter et remonte sur la paroi du flacon. Après leur formation, les chrysalides sont récoltées, dénombrées et placées dans des boîtes transparentes de $500 \mathrm{ml}$ pour émergence.

\section{Effet de la température sur la ponte et le taux d'éclosion des œufs}

Cent (100) adultes dont 50 mâles et 50 femelles de chaque biotype ont été introduits par couple dans des boîtes de ponte de $30 \mathrm{ml}$ et placés dans le bio incubateur dans les conditions de températures suivantes : $20^{\circ}, 25$ $\circ, 30^{\circ}, 32^{\circ}$ et $34^{\circ} \mathrm{C}$. Un bac en plastique de capacité 3 litres est rempli d'eau et déposé à l'intérieur de l'incubateur comme source d'humidité. Après quatre nuits d'accouplement, les pontes des femelles sont alors recueillies par jour dans des tubes de ponte de $30 \mathrm{ml}$. Les œufs pondus sont dénombrés sous une loupe et mis en incubation dans les mêmes conditions que les adultes. Pour chaque température, l'expérience a été répétée quatre (4) fois.

\section{Effet de la température sur la durée de vie} des adultes

La longévité de cinquante (50) couples placés en accouplement a été suivie et consignée tous les jours jusqu'à la mort du dernier individu. Ces adultes ont été nourris à l'aide du coton imbibé d'eau sucrée à $10 \%$.

\section{Analyse des données}

L'analyse de variance a été effectuée avec le logiciel SAS version 9.1. La séparation des moyennes a été faite par le test de Student-Newman-Keuls (SNK) au seuil de probabilité de 5\%, lorsque l'analyse de variance était significative.

\section{RESULTATS}

\section{Influence de la température sur le taux de} mortalité larvaire et émergence des adultes

Les mortalités larvaires et le taux d'émergence ne sont pas différents d'un biotype à un autre et ce, à toutes les températures testées (Tableau 1). C'est la même tendance pour le taux d'émergence des adultes d'un biotype à un autre. En revanche, le taux des émergences des adultes varie significativement avec les températures. Ainsi, lorsque la température augmente, le nombre moyen des individus qui émergent diminue pour les deux biotypes (Tableau 1).

Les durées de développement larvaire sont influencées par la température. Ainsi, le cycle de développement devient court quand la température augmente et s'allonge quand la température diminue (Tableau 2). A température égale, la durée de développement larvaire ne varie pas significativement d'un biotype à un autre (Tableau 2). C'est la même tendance qui est observée avec les durées totales de développement (larve-adulte). 


\section{Influence de la température sur la fécondité et le taux d'éclosion des œufs}

Les femelles des deux biotypes ont pondu à toutes les températures testées. Cependant, pour les températures comprises entre 20 et $32{ }^{\circ} \mathrm{C}$ les femelles du biotype du Burkina ont pondu plus d'œufs que celles du biotype témoin $(\mathrm{P}<0,0001)$. La plus forte ponte moyenne est obtenue avec le biotype du Burkina à $25^{\circ} \mathrm{C}$ et à $30^{\circ} \mathrm{C}$ tandis qu'elle est intervenue à $25^{\circ} \mathrm{C}$ chez le biotype témoin. $\mathrm{La}$ plus faible ponte est intervenue à $20{ }^{\circ} \mathrm{C}$ pour les deux biotypes (Tableau 3).

Concernant l'eclosion, tous les œufs de $M$. vitrata ont éclos aux températures testées avec une tendance à la baisse lorsque la température augmente (Tableau 3). L'éclosion des œufs du biotype du Burkina était significativement plus élévée que celle des oeufs du biotype temoin à $30{ }^{\circ} \mathrm{C}(\mathrm{F}=12,42$; $\mathrm{P}=0,0078)$ et à $34{ }^{\circ} \mathrm{C}(\mathrm{F}=35,39 ; \mathrm{P}=0,0003)$. Le plus fort taux d'éclosion des œufs a été enregistré à $25{ }^{\circ} \mathrm{C}$ tandis que la plus faible éclosion est intervenue à $34{ }^{\circ} \mathrm{C}$ pour les deux biotypes (Tableau 3).

\section{Influence de la température sur la durée de vie des adultes}

Le tableau 4 montre que la durée de vie des adultes augmente de 20 à $25^{\circ} \mathrm{C}$. A partir de cette température, elle diminue lorsque la température augmente. Il n'y a pas de différence significative entre les durées de vie des individus mâles des deux biotypes entre 20 et $32{ }^{\circ} \mathrm{C}$.

Cependant, il y a une différence significative entre la longévité des adultes mâles du biotype du Burkina et celle du biotype témoin seulement à $34{ }^{\circ} \mathrm{C}$. Chez les femelles, on note une différence significative ( $\mathrm{F}=47,38 ; \mathrm{P}<0,0001$ et $\mathrm{F}=89,91 ; \mathrm{P}<0,0001)$ entre les deux biotypes à 30,32 et à $34{ }^{\circ} \mathrm{C}$. Les femelles du biotype du Burkina ont une durée de vie plus longue que celles du biotype témoin. Mais de façon générale, pour les deux biotypes, on note que les femelles vivent plus longtemps à des températures allant de $30^{\circ} \mathrm{C}$ à $34{ }^{\circ} \mathrm{C}$.

Tableau 1 : Taux de mortalité larvaire et d'émergence des adultes de $M$. vitrata.

\begin{tabular}{cccc}
\hline Températures $\left({ }^{\circ} \mathbf{C}\right)$ & Biotype du Burkina & Témoin & Probabilité \\
\hline & \multicolumn{2}{c}{ Mortalité larvaire $(\%)$} & \\
20 & $11,3 \pm 0,9$ & $11 \pm 0,8$ & $\mathrm{P}=0,70$ \\
30 & $9 \pm 1,8$ & $9,5 \pm 1,3$ & $\mathrm{P}=0,67$ \\
32 & $9,8 \pm 1,7$ & $10,3 \pm 0,9$ & $\mathrm{P}=0,62$ \\
34 & $10,5 \pm 1,3$ & $10,3 \pm 1,3$ & $\mathrm{P}=0,79$ \\
& $11,5 \pm 1,4$ & $11,3 \pm 0,9$ & $\mathrm{P}=0,76$ \\
20 & \multicolumn{2}{c}{ Emergence $(\%)$} & \\
25 & $68,3 \pm 9,5 \mathrm{~B}$ & $68,8 \pm 9,1 \mathrm{~B}$ & $\mathrm{P}=0,94$ \\
30 & $91,5 \pm 3,4 \mathrm{~A}$ & $91 \pm 2,6 \mathrm{~A}$ & $\mathrm{P}=0,82$ \\
32 & $80,5 \pm 3,1 \mathrm{~B}$ & $79,5 \pm 1,7 \mathrm{~B}$ & $\mathrm{P}=0,89$ \\
34 & $72,3 \pm 10 \mathrm{~B}$ & $71 \pm 6,5 \mathrm{~B}$ & $\mathrm{P}=0,84$ \\
\hline
\end{tabular}

Les moyennes (土écart type) affectées des mêmes lettres en minuscule sur la même ligne ne diffèrent pas significativement au seuil de $5 \%$. 
Tableau 2 : Durée de développement larvaire.

\begin{tabular}{llcl}
\hline Températures $\left({ }^{\circ} \mathbf{C}\right)$ & Témoin & Biotype du Burkina & Probabilité \\
\hline \multirow{3}{*}{20} & Durée de développement des stades larvaires & \\
25 & $14,87 \pm 0,55$ & $14,74 \pm 0,43$ & $\mathrm{P}=1$ \\
30 & $12,28 \pm 0,58$ & $12,25 \pm 0,42$ & $\mathrm{P}=1$ \\
32 & $10 \pm 0$ & $9,76 \pm 0,42$ & $\mathrm{P}=1$ \\
34 & $8,96 \pm 0,73$ & $9,22 \pm 0,83$ & $\mathrm{P}=1$ \\
& $8,75 \pm 0,84$ & $\mathrm{P}=1$ \\
20 & Durée de développement $(1$ larve-adulte $)$ & \\
25 & $29,41 \pm 0,63$ & $29,26 \pm 0,43$ & \\
30 & $21,78 \pm 0,99$ & $21,76 \pm 0,83$ & \\
32 & $16,98 \pm 0,70$ & $16,89 \pm 1,05$ & \\
34 & $15,75 \pm 0,43$ & $15,73 \pm 0,44$ & \\
\hline
\end{tabular}

Les moyennes ( \pm écart type) affectées des mêmes lettres en minuscule sur la même ligne ne diffèrent pas significativement au seuil de $5 \%$.

Tableau 3 : Ponte moyenne journalière/femelle et taux d'éclosion des œufs.

\begin{tabular}{|c|c|c|c|}
\hline Température $\left({ }^{\circ} \mathrm{C}\right)$ & Témoin & Biotype du Burkina & Probabilité \\
\hline \multicolumn{4}{|c|}{ Ponte } \\
\hline 20 & $1,87 \pm 0,51$ & $2,85 \pm 1,61$ & $\mathrm{~F}=22,39 ; \mathrm{P}<0,0001$ \\
\hline 25 & $6,58 \pm 2,96$ & $7,74 \pm 2,74$ & $\mathrm{~F}=22,06 ; \mathrm{P}<0,0001$ \\
\hline 30 & $5,75 \pm 2,76$ & $7,73 \pm 2,91$ & $\mathrm{~F}=27,81 ; \mathrm{P}<0,0001$ \\
\hline 32 & $5,30 \pm 2,65$ & $6,70 \pm 2,20$ & $\mathrm{~F}=29,70 ; \mathrm{P}<0,0001$ \\
\hline 34 & $2 \pm 0,41$ & $4,95 \pm 3,08$ & $\mathrm{~F}=8,14 ; \mathrm{P}=0,012$ \\
\hline \multicolumn{4}{|c|}{ Eclosion (\%) } \\
\hline 20 & $33,2 \pm 1,59 \mathrm{a}$ & $35,3 \pm 1,51 \mathrm{a}$ & $\mathrm{P}=0,36$ \\
\hline 25 & $77,7 \pm 2,10 \mathrm{a}$ & $77,8 \pm 3,47 \mathrm{a}$ & $\mathrm{P}=0,38$ \\
\hline 30 & $21,5 \pm 1,5 b$ & $31,3 \pm 1,79 a$ & $\mathrm{~F}=12,42 ; \mathrm{P}=0,0078$ \\
\hline 32 & $15,2 \pm 1,31 \mathrm{a}$ & $15,6 \pm 6,3 a$ & $\mathrm{P}=0,80$ \\
\hline 34 & $5,1 \pm 0,62 b$ & $10,2 \pm 0,60 \mathrm{a}$ & $\mathrm{F}=35,39 ; \mathrm{P}=0,0003$ \\
\hline
\end{tabular}


Tableau 4 : Durée de vie (en jours) des adultes de M. vitrata.

\begin{tabular}{llcc}
\hline Températures $\left({ }^{\circ} \mathbf{C}\right)$ & Témoin & Biotype du Burkina & Probabilité \\
\hline & & Mâles & \\
20 & $13,69 \pm 1,32$ & $13,77 \pm 1,16$ & $\mathrm{P}=0,78$ \\
25 & $15,88 \pm 3,90$ & $14,77 \pm 4,84$ & $\mathrm{P}=0,26$ \\
30 & $9,14 \pm 3,30$ & $9,50 \pm 3,38$ & $\mathrm{P}=0,62$ \\
32 & $6,38 \pm 2,41$ & $6,24 \pm 1,38$ & $\mathrm{P}=0,76$ \\
34 & $5,44 \pm 1,67 \mathrm{~b}$ & $6,16 \pm 2,12 \mathrm{a}$ & $\mathrm{F}=5,68 ; \mathrm{P}=0,02$ \\
& & Femelles & \\
20 & $14,60 \pm 3,97$ & $15,04 \pm 4,56$ & $\mathrm{P}=0,63$ \\
30 & $12,26 \pm 3,92$ & $13,13 \pm 2,17$ & $\mathrm{P}=0,42$ \\
32 & $10,62 \pm 3,18 \mathrm{~b}$ & $12,45 \pm 2,75 \mathrm{a}$ & $\mathrm{F}=47,38 ; \mathrm{P}<0,0001$ \\
34 & $8,20 \pm 2,72 \mathrm{~b}$ & $12,30 \pm 3,35 \mathrm{a}$ & $\mathrm{F}=5,47 ; \mathrm{P}=0,02$ \\
& $6,03 \pm 2,16 \mathrm{~b}$ & $9,86 \pm 2,06 \mathrm{a}$ & $\mathrm{F}=89,91 ; \mathrm{P}<0,0001$
\end{tabular}

Les moyennes ( \pm écart type) affectées des mêmes lettres en minuscule sur la même ligne ne diffèrent pas significativement au seuil de $5 \%$.

\section{DISCUSSION}

Les larves se sont développées et ont bouclé leur cycle à toutes les températures évaluées. Ce résultat est contraire à ceux d'une étude similaire menée par Jackai et Inang (1992) qui notent que le développement jusqu'au stade adulte est complété seulement à $22{ }^{\circ} \mathrm{C}$ et $28{ }^{\circ} \mathrm{C}$. Nous ignorons les vraies raisons même si les conditions de manipulations pourraient en être une, en occurrence la nature du substrat alimentaire utilisé mais aussi le comportement des larves dans celui-ci. En effet les larves à partir du second stade, pénètrent en profondeur dans le substrat alimentaire. Dans ces conditions, elles ne subissent pas directement l'effet de la température qui règne dans l'incubateur et qui peut affecter leur développement. Ce n'est qu'à partir du dernier stade larvaire qu'une grande partie des larves ressortent du substrat alimentaire pour se «nymphoser» sur les parois des boîtes, subissant ainsi l'effet direct de la température qui règne dans l'incubateur d'où la mortalité nymphale et le taux d'émergence observés. Le fort taux de formation des chrysalides justifie celui des émergences observées à $25^{\circ} \mathrm{C}$, suggérant de fait que c'est la température convenable au développement optimal de l'insecte.

Les températures ont affecté le développement larvaire. Ainsi, le cycle de développement est plus long à des températures basses et devient court à des températures élevées. Plusieurs auteurs DU et al. (2012) sur Conogethes punctiferalis ; Tang et al. (2016) sur Beet Webworm; Nakamura et al. (2017) sur Lasius japonicus, Nakanishi et al. (2017) sur Cossus insularis, ont rapporté que l'augmentation des températures avait un effet réductif sur le développement larvaire des insectes, montrant ainsi que la température est un facteur environnemental de premier plan de régulation de développement. L'absence de différence entre les deux biotypes serait due à l'effet de la nature du substrat alimentaire qui réduirait l'impact direct de la température sur les larves.

Ces travaux montrent que $M$. vitrata est capable de vivre à des températures extrêmes $\left(20{ }^{\circ} \mathrm{C}\right.$ et $\left.34{ }^{\circ} \mathrm{C}\right)$ avec des taux de survie larvaire et d'émergence imaginale qui dépassent respectivement $80 \%$ et $70 \%$. Ce taux de survie est nettement supérieur à celui obtenu par Adati et al. (2004). Cependant, la 
collecte des larves sur les plantes hôtes sauvages après le mois de décembre à un moment où le taux moyen d'humidité est d'environ $19 \%$ couplé à de hautes températures, nous permet d'émettre une hypothèse, celle de l'acquisition par l'insecte des capacités lui permettant de supporter ces températures. Cette capacité proviendrait, à notre avis, d'une probable adaptation des populations de l'insecte à cet environnement peu humide. L'insecte se serait ainsi adapté à la faible humidité et aux hautes températures, au cours de sa dispersion, avec le niébé, vers l'Afrique de l'Ouest et la zone sahélienne.

La ponte a augmenté pour atteindre son pic à $25{ }^{\circ} \mathrm{C}$ suggérant que celle-ci est une température convenable à l'insecte. Jackai et al. (1990) ont rapporté que la femelle de $M$. vitrata préfère s'accoupler et pondre dans les conditions de basses températures et de forte humidité. La ponte a diminué avec l'augmentation de la température. Cette diminution liée à l'augmentation de la température est rapportée par Chi et al. (2005) qui ont obtenu une moyenne de ponte journalière inversement corrélée avec la température $\left(27^{\circ}\right.$ à $\left.20^{\circ} \mathrm{C}\right)$, même si les températures testées par ces auteurs sont faibles comparativement aux nôtres. En plus, nos résultats ont montré une forte capacité de ponte à $30^{\circ} \mathrm{C}$ couplée à un taux d'éclosion également élevé à $25^{\circ}$ et $30^{\circ} \mathrm{C}$. Des résultats similaires ont été rapportés par Ichikawa et al. (2017) sur les œufs de Pantala flavescens qui ont tous éclos jusqu'à des températures allant à $35^{\circ} \mathrm{C}$. Mais à $34^{\circ} \mathrm{C}$ le taux d'éclosion qui a été de $10.2 \%$ était plus faible comme l'a signalé Adati et al. (2004). En effet, selon ces derniers, à $34.3{ }^{\circ} \mathrm{C}$, les taux de survie des œufs de M. vitrata sont de moins de $10 \%$.

La longévité des adultes diminue lorsque la température augmente, avec des femelles qui vivent plus longtemps que les mâles. L'ensemble de ces résultats peuvent se comprendre si on admet qu'ils sont la conséquence de l'hypothèse formulée, celle d'une possible adaptation.

De tous ces résultats, il ressort que les paramètres biologiques mesurés ont exprimé leur meilleur potentiel à $25^{\circ} \mathrm{C}$ suggérant ainsi que cette température est la plus favorable au développement de l'insecte.

\section{Conclusion}

Les résultats de cette étude montrent que $M$. vitrata supporte aussi bien les basses températures que les températures relativement élevées. Elle a aussi montré que $25{ }^{\circ} \mathrm{C}$ est la température idéale de développement de l'insecte. La longévité, la fécondité et la durée d'incubation des œufs obtenues à cette température militent en faveur de cette conclusion. En plus, la ponte obtenue à la température de $30{ }^{\circ} \mathrm{C}$ chez le biotype du Burkina et les meilleures performances que celle-ci présente comparativement au biotype témoin nous permettent de dire que l'insecte connu pour être celui des zones humides, est capable de vivre et de se multiplier dans un environnement peu humide. Les résultats seront utiles pour prévoir l'incidence des ravageurs dans les cultures de niébé des zones de savane ouest-africaine.

\section{CONFLIT D'INTERETS}

Il n'y aucun conflit d'intérêt entre les auteurs de cet article.

\section{CONTRIBUTIONS DES AUTEURS}

FT est l'investigateur principal et concepteur du protocole ; WA a contribué à la rédaction du manuscrit; $\mathrm{DC}$ et $\mathrm{SA}$ ont supervisé le travail.

\section{REMERCIEMENTS}

$\mathrm{Au}$ terme de cette étude, nos remerciements vont à l'adresse du technicien BAMA Hervé qui pendant deux (02) mois nous a apporté son assistance technique. Nos remerciements vont également à l'endroit de Dr TAMÒ Manuele, représentant résidant de l'Institut International d'Agriculture Tropical (IITA) pour son appui logistique et ses précieux conseils.

\section{REFERENCES}

Adati T, Nakamura S, Tamò, Kawazu K. 2004. Effect of temperature on development and survival of the legume 
pod borer, Maruca vitrata (Fabricius) (Lepidoptera: Pyralidae) reared on a semi-synthetic diet. Appl. Entomol. Zool. (Jpn), 39(1): 139-145. DOI: https://doi.org/10.1303/aez.2004.139

Adati T, Tamò M, Koji S, Downham MCA. 2013. Effect of migration and mating status of the legume pod borer, Maruca vitrata (Fabricius) (Lepidoptera: Crambidae) on the efficacy of synthetic sex pheromone traps in West Africa. In Proceedings of the fifth World Cowpea Conference, Boukar O, Coulibaly O, Fatokun C, Lopez K, Tamò M (eds). IITA, Ibadan, Nigeria; 260-272.

Atachi P, Dannon AE, Arodokoun DY, Tamò M. 2002. Distribution and sampling of Maruca vitrata (FABRICIUS) (Lep., Pyralidae) larve on Lonchocarpus sericeus (PÜIR) H. B. and K. J Appl. Ent., 126: 188-193. DOI : https://doi.org/10.1046/j.14390418.2002.00633.x

Atachi P. 1998. Etude bioécologique de Maruca testulalis (Geyer) dans les cultures de Vigna unguiculata (L.) en République du Bénin : perspectives de lutte intégrée. Thèse de Doctorat ès Sciences Naturelles, Faculté des Sciences et Techniques de 1'Université d'Abidjan (Cocody), Abidjan, p. 351.

Ba NM, Margam VM, Dabiré LC, Sanon A, McNeil JN, Murdock LL, Pittendrigh BR. 2009. Seasonal and regional distribution of the cowpea pod borer Maruca vitrata (Lepidoptera: Crambidae) in Burkina Faso. Int J Trop Insect Sci., 29(3): 109-113. DOI : https://doi.org/10.1017/S1742758409990 $21 \mathrm{X}$

Chi Y, Sakamaki Y, Tsuda K, Kusigemati K. 2005. Effect of Temperature on Oviposition and Adult Longevity of the Legume Pod Borer, Maruca vitrata (Fabricius) (Lepidoptera: Crambidae). Kagoshima. Jpn. J. Appl. Entomol. Zool., 49: 29-32.

DOI : https://doi.org/10.1303/jjaez.2005.29

Dabiré CL. 2001. Etude de quelques paramètres biologiques et écologiques de
STAL., 1855 (Hemiptera : Coreidae), punaises suceuses de gousses du niébé Vigna unguiculata (L) WALP. dans une perspective de lutte durable contre l'insecte au Burkina Faso. Thèse de Doctorat d'état, Université de Cocody, Abidjan, p. 179.

Downham MCA, Hall DR, Chamberlain DJ, Cork A, Farman DI, Tamò M, Dahounto D, Datinon B, Adetonah S. 2003. Minor components in the sex pheromone of legume pod borer: Maruca vitrata development of an attractive blend. $J$. Chem. Ecol., 29(4): 989-1011. DOI: 10.1023/A: 1022996103147.

Du YL, Guo HM, Sun SL, Zhang MZ, Zhang AH, Wang JB, Qin L. 2012. Effects of temperature on the development and reproduction of the yellow peach moth, Conogethes punctiferalis (Lepidoptera: Pyralidae) [J]. Acta Entomol. Sin., 55(5) : 561-569.

FAOSTAT. 2013. Production de niébé, superficie et rendements en Afrique Subsaharienne (ASS, 2011 -2013). Source : FAOSTAT (www.faostat.org). DOI :

https://link.springer.com/article/10.1007/ s13355-016-0460-1.

Ichikawa Y, Yokoi T, Watanabe M. 2017. Thermal factors affecting egg development in the wandering glider dragonfly, Pantala flavescens (Odonata: Libellulidae). Appl. Entomol. Zool. (Jpn.), 52(1) : 89-95. DOI: https://link.springer.com/article/10.1007/ s13355-016-0457-9.

Jackai LEN, Inang EE, Nwobi P. 1992. The potential for controlling postflowering pests of cowpea, Vigna unguiculata WALP. using neem, Azadirachta indica A. mss, IITA, Oyo Road, Ibadan, Nigeria. Trop. Pest Manag., 38(1) : 5660.

DOI : https://doi.org/10.1080/09670879209371 646

Jackai LEN, Ochieng RS, Raulston JR. 1990. Mating and oviposition behavior of the legume pod borer, Maruca testulalis. Entomol. Exp. et Appl., 59(2) : 179-186. 
DOI : https://doi.org/10.1111/j.15707458.1990.tb01395.x

Jackai LEN, Raulston JR. 1988. Rearing the legume pod borer, Maruca testulalis Geyer (Lepidoptera: Pyralidae) on artificial diet. Trop. Pest Manag., 34(2) : 168-172. DOI : https://doi.org/10.1080/09670878809371 236

Kabore KH. 2013. Effet de microdosage de la fumure organo-minerale sur la dynamique de Macrophomina phaseolina (Tassi) Goid., agent causal de la pourriture charbonneuse du niébé. Memoire de DEA, Université de Ouagadougou, Ouagadougou, p. 35.

Kadri A, Zakari Moussa O, Sidoyacouba A, Hame Abdou KK, Karimoune L. 2013. Gestion intégrée de Maruca vitrata (FABRICIUS, 1787) et Megalurothrips sjostedti (TRYBOM, 1908), deux insectes ravageurs majeurs du niébé au Niger. Int. J. Biol. Chem. Sci., 7(6): 2549-2557. DOI: 10.4314/ijbcs.v7i6.29.

Nakamura K, Fujiyama M, Ohta K. 2017. Effect of temperature on queen oviposition and seasonal colony development in Lasius japonicus (Hymenoptera: Formicidae). Appl. Entomol. Zool. (Jpn.), 52(1): 107-112.

Nakanishi T, Kaneda T, Nakamuta K. 2017. Effects of temperature on the development and circannual control of pupation in the carpenter moth, Cossus insularis (Lepidoptera: Cossidae), reared on an artificial diet. Appl. Entomol. Zool. (Jpn.), 52(1): 29-35. DOI : https://link.springer.com/article/10.1007/ s13355-016-0450-3.

Sanginga N, Bergvinson D. 2015. Oléagineux et protéagineux. $26 \mathrm{p}$.

Singh SR. Jackai LEN. 1985. Insect pest of cowpea in Africa: Their life cycle, economic and potential for control. In
Cowpea Research, Production and utilization, Singh SR et Rachie KO (eds). London, John Wiley, 217-231.

Steigenga MJ, Fischer K. 2009. Fitness consequences of variation in developmental temperature in a butterfly. J. Therm. Biol. 34(5) : 244-249. DOI: https://doi.org/10.1016/j.jtherbio.2009.0 3.001 .

Tang J, Cheng Y, Sappington TW, Jiang X, Zhang L, Luo L. 2016. Egg Hatch and Survival and Development of Beet Webworm (Lepidoptera: Crambidae) Larvae at Different Combinations of Temperature and Relative Humidity. $J$. Econ. Entomol., 109(4) : 1603-1611. DOI: https://doi.org/10.1093/jee/tow106.

Traoré F, Ba NM, Dabiré-Binso CL, Sanon A, Pittendrigh BR. 2014. Annual cycle of the legume pod borer Maruca vitrata Fab. (Lepidoptera: Crambidae) in Southwestern Burkina Faso. ArthropodPlant Interact., 8(2) : 155-162.

Traoré F, Dabiré-Binso CL, Ba NM, Sanon A, Pittendrigh BR. 2013. Feeding preferences of the legume pod borer Maruca vitrata (Lepidoptera: Crambidae) larvae and suitability of different flower parts for larval development. Int. .J Trop. Insect Sci., 33(2) : $\quad 107-113 . \quad$ DOI : https://doi.org/10.1017/S1742758413000 088.

Traore F. 2014 : Étude de la bio-écologie de Maruca vitrata Fabricius (Lepidoptera : Crambidae) foreuse des gousses de niébé (Vigna unguiculata (L.) Walp.) dans une perspective de gestion durable du ravageur au Burkina Faso. Mémoire de thèse unique, Université de Ouagadougou, Ouagadougou, p. 131. 